\title{
NORM INEQUALITIES FOR SOME SINGULAR INTEGRAL OPERATORS
}

\author{
TAKAHIKO NAKAZI
}

\begin{abstract}
Let $\mathscr{B}$ be a von Neumann algebra and $P$ a selfdjoint projection. For $A$ and $B$ in $\mathscr{B}$, set $S_{A, B}=A P+B Q$ where $Q=I-P$. The operator $S_{A, B}$ will be called a singular integral operator. When $\mathscr{B}=L^{\infty}(T)$ where $L^{\infty}(T)$ is the usual Lebesgue space on the unit circle and $P$ is an analytic projection, in [6] we established formulae for norms of $S_{A, B}$ and $\left(S_{A, B}\right)^{-1}$. In this paper, if $\mathscr{A}=\{D \in \mathscr{B}: P D P=D P\}$ and $(\mathscr{B}, \mathscr{A}, P)$ has a lifting property, then we will establish formulae of norms of $S_{A, B}$ and $\left(S_{A, B}\right)^{-1}$. These formulae are operator theoretic and different from the previous ones. There are several examples such that $(\mathscr{B}, \mathscr{A}, P)$ has a lifting property. As result, we give several interesting inequalities.
\end{abstract}

Mathematics subject classification (1991): 45E10, 47B 35, 46J15, 46K50.

Key words and phrases: Singular integral operator, norm, analytic operator algebra, lifting theorem.

\section{REFERENCES}

[1] M. Cotlar AND C. SADOSKY, On the Helson-Szegö theorem and a related class of modefied Toeplitz kernels, Proc. Sympos. Pure Math., Vol.35, Amer. Math. Soc., Providence, R.I., 1979, 383-407.

[2] A. Devinatz AND M. ShInBROT, General Wiener-Hopf operators, Trans. Amer. Math. Soc., 145 (1969), 467-494.

[3] R. G. Douglas, Banach Algebra Techniques in Operator Theory, Academic Press, New York, 1972.

[4] I. Gohberg AND N. KRUPNIK, One-Dimensional Linear Singular Integral Equations, Vols. I, II, Birkhäueser Verlag, Basel, 1992.

[5] T. NAKAZI, A lifting theorem and analytic operator algebras, Proc. Amer. Math. Soc., 104 (1988), 1081-1085.

[6] T. NAKAZI AND T. YAMAMOTO, Norms of some singular integral operators, J. Operator Th. 40 (1998), 187-207.

[7] M. SHINBROT, On singular integral operators, J. Math. Mechanics, 13 (1964), 587-601. 